\title{
Whammies and double whammies: The effect of length on nonword reading
}

\author{
KATHLEEN RASTLE and MAX COLTHEART \\ Macquarie University, Sydney, New South Wales, Australia
}

\begin{abstract}
In the work presented here, the length effect in nonword reading aloud was investigated in order to assess whether that effect is driven by the number of letters in a string or by the number of graphemes in a string. Simulation work with the Dual-Route Cascaded (DRC) model (e.g., Coltheart, Curtis, Atkins, \& Haller, 1993; Coltheart \& Rastle, 1994) uncovered a surprising finding regarding the length effect; the same result was obtained in an experiment with human subjects. The results are discussed in terms of the DRC model, with particular reference to serial processing and interphoneme inhibition, two properties critical to understanding the effect reported here.
\end{abstract}

It is well known that naming latencies for words and nonwords are affected by string length: naming latency increases as string length increases (Frederiksen \& Kroll, 1976; Weekes, 1997; for a review, see Henderson, 1982). For words, this length effect is modulated by word frequency; low-frequency words show a larger effect of length than do high-frequency words (Content \& Peereman, 1992). Weekes (1997) has further suggested that the size of this length effect is affected by the lexical status of the string; nonwords show a much larger effect of length than do words.

Weekes (1997) argued that the interaction between length and lexicality on naming latency is evidence against any model that processes words and nonwords via a single mechanism (e.g., Plaut, McClelland, Seidenberg, \& Patterson, 1996) and that this evidence is more coherently explained by the Dual-Route Cascaded (DRC) model of reading (Coltheart, Curtis, Atkins, \& Haller, 1993; Coltheart \& Rastle, 1994). The DRC model translates orthography to phonology via two procedures: a lexical procedure that operates in parallel across the input string and a nonlexical procedure that operates serially from left to right across the string. Input from these two routes contributes to the rise of activation in a common phoneme system to generate a pronunciation.

Length effects on naming latency in the DRC model reflect the serial operation of the nonlexical route; the extent to which the nonlexical route is involved in processing therefore determines the size of the length effect for any stimulus. Because the lexical route processes highfrequency words so quickly, the nonlexical route makes litthe or no contribution to the naming of these words. When

We are grateful to Dave Balota, Derek Besner, and Ken Paap for helpful suggestions regarding an earlier version of this article. We are similarly grateful to Dave Plaut for running simulations on the attractor network, and to Jo Ziegler and Conrad Perry for helpful and critical discussion. Correspondence should be addressed to Kathleen Rastle, Macquarie University, Department of Psychology, Sydney, NSW 2109, Australia (e-mail: kathy@currawong.bhs.mq.edu.au). the stimulus is a low-frequency word, however, lexical processing is sufficiently slow to allow for a substantial contribution from the nonlexical route (this is why irregular words have longer naming latencies than regular words, but only when those words are of low frequency, e.g., Paap \& Noel, 1991; Seidenberg, Waters, Barnes, \& Tanenhaus, 1984). When the stimulus is a nonword, the nonlexical route is the major determinant of pronunciation, since nonwords cannot be pronounced correctly via the lexical route. It follows obviously from the DRC account of reading aloud that the length effect on naming latency should be smaller for high-frequency words than for low-frequency words, and smaller for low-frequency words than for nonwords. As we have noted, this is what has been reported.

A subtle, but important, aspect of the length effect remains unexplored, however. The nonlexical route of the DRC model applies rules serially, from left to right, across a letter string. These rules could be applied serially as letters are submitted for translation or as graphemes are submitted for translation (where, by "grapheme," we mean the written representation of a phoneme). In the former case, one would expect that the DRC model's nonword naming latency would depend on the number of letters in the input string; in the latter case, one would expect that these latencies would depend on the number of graphemes in the input string. Normally, there is a high correlation between the number of graphemes in a nonword and the number of letters in a nonword - this correlation was +.53 in the stimuli used by Weekes (1997) - and so determining which variable is critical to the length effect is not straightforward.

However, it is possible to disentangle these variables, and the experiments we report were designed to do so. We chose two sets of five-letter nonwords, one set containing three graphemes (e.g., FOOCE) and another set containing five graphemes (e.g., FRULS), matched on a number of properties. If the number of graphemes-and not the number of letters-is implicated in serial processing, then those nonwords with few graphemes (e.g., FOOCE) 
should be named more quickly than those nonwords with many graphemes (e.g., FRULS). If, instead, it is the number of letters that is implicated in serial processing, then there should be no latency difference between the two sets of nonwords, since all items in each set are exactly five letters long.

Whether the unit of serial operation is the grapheme or the letter is critical empirically and theoretically. In particular, the outcome of this experiment will determine whether the nonlexical route of the DRC model should operate letter by letter or grapheme by grapheme.

To explore the straightforward predictions described above, two versions of the DRC model were compared. In the DRC-G model, the nonlexical route translates the string grapheme by grapheme. ${ }^{1}$ In the DRC-L model (the original model reported by Coltheart et al., 1993, and Coltheart \& Rastle, 1994), the nonlexical route translates the string letter by letter.

In simulations using the DRC-G model, then, we expected that nonword naming latency would increase with number of graphemes but would be unaffected by number of letters when number of graphemes was held constant (e.g., the FOOCE items would be named faster than the FRULS items). In contrast, in simulations using the DRC-L model, we expected that nonword naming latency would increase with number of letters, but would be unaffected by number of graphemes when number of letters was held constant (e.g., there would be no difference between FOOCE items and FRULS items).

\section{EXPERIMENT 1 DRC Simulation}

\begin{abstract}
Method
Stimuli. Two lists of 24 five-letter nonwords were devised. One set of nonwords contained three graphemes; the other set contained five graphemes. The lists were pairwise matched as closely as possible on initial phoneme, number of neighbors, number of body friends, and the summed frequency of body friends. None of the nonwords had any body enemies. The stimuli are contained in the Appendix.

Parameter set. Prior simulation work with the original DRC model (DRC-L) has isolated a parameter set with which the model simulates a number of effects in reading aloud and reads exception words and nonwords extremely well. Among the effects we have simulated with this parameter set are the regularity effect and its interaction with frequency (e.g., Seidenberg et al., 1984), the position of irregularity effect strategic effects in reading, and the effects of speeded and unspeeded naming (Rastle \& Coltheart, in press). This set of parameters was also used in both of the simulations reported here.

Procedure. The 48 nonword items were submitted to both versions of the DRC model, and reaction times (in processing cycles) were recorded. Because we were not interested in comparing overall performance of the models, data from each model were analyzed separately, with the errors (and their matched items) produced by each model removed from the respective analysis. The DRC-G model produced two errors: BOACE was pronounced as "BOTH", and SERCE was pronounced as "SERK." The DRC-L model did not produce any errors.
\end{abstract}

\section{Results and Discussion}

As shown in Table 1, the DRC-G model behaved according to prediction; a repeated measures analysis of variance (ANOVA) revealed that nonwords with three
Table 1

DRC and Human Naming Latency as a Function of Nonword Grapheme Condition

\begin{tabular}{|c|c|c|c|c|}
\hline \multicolumn{5}{|c|}{ Nonword Grapneme Conaition } \\
\hline & $\begin{array}{l}\text { DRC-G } \\
\text { (cycles) }\end{array}$ & $\begin{array}{l}\text { DRC-L } \\
\text { (cycles) }\end{array}$ & $\begin{array}{c}\text { Human Subject } \\
\text { Data (msec) }\end{array}$ & $\begin{array}{c}\text { Item } \\
\text { Data (msec) }\end{array}$ \\
\hline 3-Grapheme Nonwords & 133 & 164 & 586 & 585 \\
\hline 5-Grapheme Nonwords & 156 & 157 & 569 & 568 \\
\hline
\end{tabular}

graphemes were named more quickly than were nonwords with five graphemes $\left[F(1,21)=67.48, M S_{\mathrm{e}}=\right.$ $84.20, p<.05]$. The DRC-L model produced results in the opposite direction: nonwords with three graphemes were named more slowly than were nonwords with five graphemes $\left[F(1,23)=8.54, M S_{\mathrm{e}}=65.64, p<.01\right]$. Item data are contained in the Appendix.

As predicted, the DRC-G model produced shorter reaction times for items with three graphemes than for items with five graphemes. The DRC-L model behaved counter to our intuition, however. Despite the fact that the two sets of items contained exactly the same number of letters - the fact that had led us to predict that this model would not exhibit naming latency differences between the two types of nonword - the DRC-L model produced longer reaction times for items with three graphemes (e.g., FOOCE) than for items with five graphemes (e.g., FRULS).

What of human subjects? Would they show the effect that the DRC-G model shows or the effect that the DRC-L model shows, or would their behavior be inconsistent with both of these models?

\section{EXPERIMENT 2 Human Subjects}

\section{Method}

Subjects. The subjects were 23 first-year psychology students from Macquarie University. All had normal or corrected-to-normal vision and all were native Australian-English speakers. They received introductory course credit for their participation.

Stimuli. The same 48 nonwords used in Experiment 1 were used here. They are listed in the Appendix.

Apparatus and Procedure. Stimulus presentation and data recording were controlled by the DMASTR software (Forster \& Forster, 1990) running on a DeltaCom $486 \mathrm{PC}$. The subjects were seated approximately 16 in. from the computer monitor and were instructed to read the nonwords as quickly and as accurately as possible. They were given 10 practice trials. The 48 nonwords were presented to subjects in random order.

\section{Results and Discussion}

Data were collected, and the reaction times for spoiled trials (because of voice-key failure) and errors (along with their matched nonwords) were discarded. The remainder of the data were winsorized to the second standard deviation boundary.

Data were analyzed both by subjects and by items. Means are given in Table 1, and full item data are contained in the Appendix. Repeated measures ANOVAs confirmed that naming latencies for nonwords with three graphemes were significantly different from naming la- 
tencies for nonwords with five graphemes, by both subjects $\left[F(1,22)=7.6, M S_{\mathrm{e}}=444, p<.05\right]$ and items $\left[F(1,23)=6.2, M S_{\mathrm{e}}=616, p<.05\right]$. However, contrary to the predictions discussed earlier, but consistent with what actually happens in the DRC-L model, the threegrapheme nonwords yielded longer naming latencies than the five-grapheme nonwords.

Error data were analyzed in the same way. Repeated measures ANOVAs confirmed that there was no effect of number of graphemes in the error data, either by subjects [three graphemes, $4.3 \%$; five graphemes, $4.2 \%$ : $F(1,22)=.03$, n.s.] or by items [three graphemes, $4.7 \%$; five graphemes, $4.2 \%: F(1,23)=.07$, n.s.].

As discussed earlier, the two possible outcomes we had foreshadowed were that there would be no difference in naming latencies between the two types of nonword (we considered that this would imply that the length effect on nonword naming latency depends on number of letters) or that the nonwords with three graphemes would have shorter naming latencies than the nonwords with five graphemes (we considered that this would imply that the length effect on nonword naming latency depends on number of graphemes). We did not initially consider the logically possible third alternative (that the nonwords with three graphemes would yield longer naming latencies than the nonwords with five graphemes) since there seemed to be no reason to expect that this could happen. Yet that is what we observed in the behavior of the DRC-L model and in the behavior of human subjects. How is this apparently paradoxical finding to be explained? It turns out that this puzzle has arisen only through a failure on our part to think deeply enough about just how the nonlexical route of the DRC model actually operates in its DRC-L (letter-by-letter) version.

The activity of the nonlexical route in the DRC model is controlled by two parameters. One parameter specifies the number of cycles elapsed before the nonlexical route begins to process the first item (currently set at 10 cycles). The other parameter specifies the number of cycles elapsed before the nonlexical route begins to process each subsequent item (currently set at 17 cycles). In these statements, "item" denotes "grapheme" for the DRC-G version of the model and "letter" for the DRC-L version of the model.

To solve the problem posed by the human data and the performance of the DRC-L model, we studied in detail how the DRC-L version of the model behaves when naming an item such as FOOPH. On Cycle 11 , nonlexical translation of the first letter $F$ occurs, and the phoneme unit $/ f /$ begins to rise in the first phoneme set. At the 28 th processing cycle, the next letter in the string, $\mathrm{O}$, becomes available for translation, making the input to the nonlexical route FO. Since the GPC rule for the grapheme $O$ is $\mathrm{O} \rightarrow / \mathrm{Q} /$, activation of the phoneme unit $/ \mathrm{Q} /$ in phoneme set two begins to rise. ${ }^{2}$

The correct second phoneme for FOOPH is not, of course, $/ \mathrm{Q} /$; it is $/ \mathrm{u} /$. The string FOo does not become available for translation by the nonlexical route until
Cycle 45, however, and it is only then that the correct phoneme $/ \mathrm{u} /$ in the second phoneme set begins to rise, guided by the application of the GPC rule OO $\rightarrow / \mathrm{u} /$.

At this point in processing time, activation of the spurious phoneme $/ \mathrm{Q} /$ has been building in the second phoneme set for the previous 17 cycles. Within any set of units in the DRC model, there is full lateral inhibition. Thus, the correct phoneme / $\mathrm{u} /$ will meet with a hostile reception: an already-active phoneme in its set will exert inhibition upon it, thus slowing the rate at which its activation rises perhaps even blocking its activation altogether. We call this effect a whammy ("whammy: a potent force or attack; specifically, a paralyzing or lethal blow"--MerriamWebster). The whammy is thus a potential mechanism for explaining why the putatively easily processed threegrapheme items such as FOOPH might instead actually suffer from their orthographic-phonological relationships.

Next, consider what happens on Cycle 61 when the fourth letter of FOOPH becomes available for GPC translation. The input to the nonlexical route is now FOOP, and so activation for $/ \mathrm{p} /$ in the third phoneme set will begin to rise. But this too is a spurious phoneme, since the correct third-position phoneme is $/ \mathbf{f} /$. Hence, when the fifth letter becomes available for translation on Cycle 78, the rise of activation for $/ \mathrm{f} /$ in the third phoneme set will also be slowed by lateral inhibition. The nonword FOOPH will thus experience an inhibition of both its second and its third phonemes. We call this effect a double whammy ("double whammy: a combination of two usually adverse forces, circumstances, or effects"-Merriam-Webster).

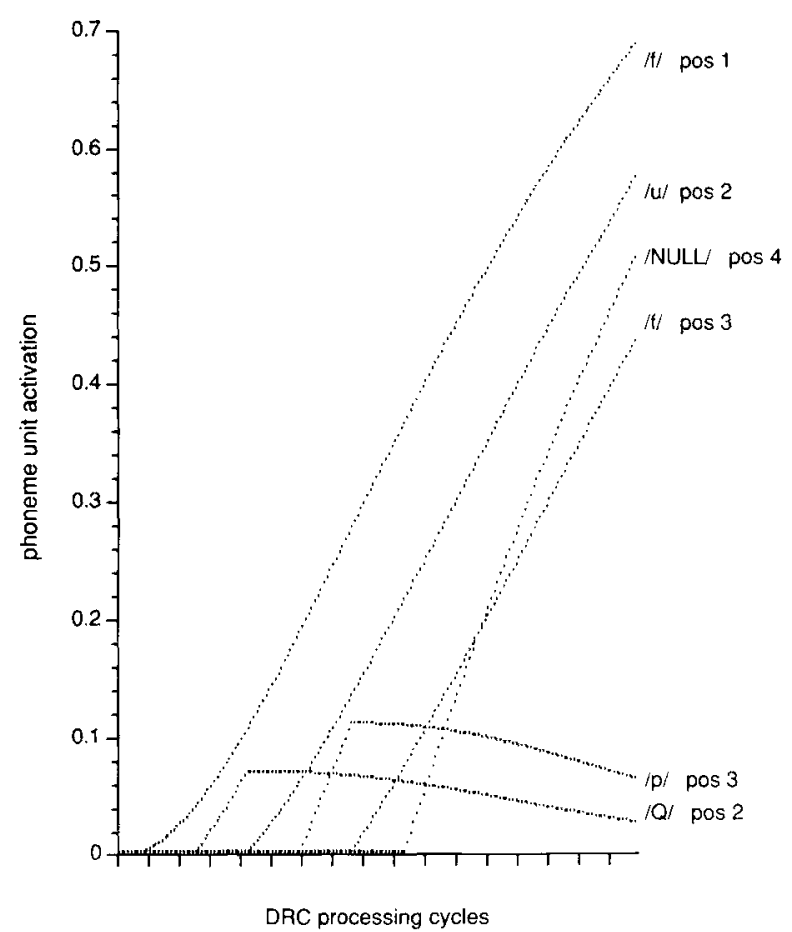

Figure 1. The DRC-L model reading FOOPH (one tick $=10$ processing cycles). 
These events are clearly visible in Figure 1, which plots the activations of the correct and spurious phonemes of FOOPH as a function of processing cycle when this item is read by the DRC-L model.

Not all multiletter graphemes are harmful to processing, however. TREFF, for example, contains the multiletter grapheme FF, but when the first four letters TREF become available for nonlexical translation, the "spurious" phoneme generated by the GPC rule " $\mathrm{F} \rightarrow / \mathrm{f} /$ " happens to be the correct phoneme, also generated when the fifth letter becomes available. The correct phoneme in Phoneme Set 4 will not encounter inhibition: on the contrary, it will encounter a warm welcome, since its phoneme unit will already be active. Other multiletter graphemes produce extremely destructive whammies, however. The correct phoneme $/ 1 /$ for grapheme EIGH, for example, will undergo enormous inhibition from several spurious phonemes allowed to rise over many processing cycles.

Clearly our original prediction regarding three grapheme nonwords was obtuse. Using the DRC-L model as an aid to thought, it is possible to see why what was once a paradoxical result reveals, instead, some remarkable subtleties in the procedures by which nonwords are read aloud. Items like FOOCE are named more slowly than items like FRULS in the model because of whammy and double whammy effects. We claim that this is also true for human readers. ${ }^{3}$

Phonological dyslexia. The whammy effect is also relevant to the form of acquired dyslexia known as phonological dyslexia, the characteristic symptom of which is a selective impairment in the ability to read nonwords. In the first investigation of phonological dyslexia (Derouesne \& Beauvois, 1979), two properties of nonwords were manipulated in a reading-aloud task involving 4 patients: pseudohomophony (accuracy of reading pseudohomophones vs. nonpseudohomophonic nonwords) and what Derouesne and Beauvois referred to as "graphemic complexity" but which we would refer to as "presence vs. absence of whammies." A double dissociation between these factors emerged: 2 patients exhibited only an effect of pseudohomophony, and 2 patients, an effect of graphemic complexity. These results provide independent evidence for our claims regarding nonword reading.

Preliminary simulation work with the DRC model has shown that substantial increases to the interletter interval parameter results in impaired nonword reading, with whammied nonwords producing a much higher error rate than nonwhammied nonwords. Increasing the value of this parameter has also been shown to simulate the pseudohomophone advantage in reading accuracy described by Derouesne and Beauvois (1979; Coltheart, Langdon, \& Haller, 1996). Thus, both of these types of phonological dyslexia can be simulated by the DRC model, although it remains to be determined whether the DRC model can simulate the double dissociation between them.

Subtle whammies. The story does not end here. Let us consider in more detail the individual DRC-simulated latencies for the five-grapheme nonwords in the Appendix. Most latencies are within a narrow band, 149-157 cycles. But there is also a group of three outliers with naming latencies between 170 and 185 cycles. Since these items have no multiletter graphemes, and so should not be whammied, what is causing them to be slowed?

It turns out that these items are, in fact, whammied also, although in particularly subtle ways. Consider the naming of TWENK. When the fourth letter, $\mathrm{N}$, becomes available to the nonlexical system, it is translated to $/ \mathrm{n} /$ and activation for this unit in the fourth phoneme set begins to rise. Not until 17 cycles later, when the fifth letter, $\mathrm{K}$, becomes available for translation, is the phonotactic rule that states "Convert $/ \mathbf{n} /$ to $/ \mathrm{N} /$ when followed by / $\mathrm{k}$ /" applied by the DRC model's nonlexical system, but, by this time, a whammy has already occurred: the rise of $/ \mathrm{N} /$ is slowed by the already active presence of $/ \mathrm{n} /$. The same type of whammy occurs in the item PRENK.

FRULS also encounters a particularly subtle whammy. When the $\mathrm{S}$ becomes available to the nonlexical system, it is translated to $/ \mathrm{s} /$, and activation for this unit in the fifth phoneme set begins to rise. Not until 17 cycles later does the nonlexical system reach the null letter character, signifying that $\mathrm{S}$ was the last letter. At this time, the phonotactic rule stating "If $/ \mathrm{s} /$ is preceded by $/ 1 /$ and is the terminal consonant, convert it to $/ \mathbf{z} / "$ is applied. However, the rise of $/ z /$ is already whammied by the rise of $/ \mathrm{s} /$.

Another serial effect. Not all whammies are the same; indeed, in the DRC-L model, the position of the whammy in a nonword has a tremendous effect on its consequences for naming. Consider the nonwords STOPH and PHOTs. In both cases, one phoneme, /f/, will be whammied (there will be competition from the spurious phoneme $/ p /$ ). Since both items have five letters, four graphemes, and one whammy, one might expect them to have exactly the same naming latency. This expectation is incorrect as far as the model is concerned, because it neglects the fact that the nonlexical procedure operates from left to right.

Letters are submitted for nonlexical translation serially in the DRC-L model, and naming does not occur until all phonemes have reached their critical activation. Thus, a last-position whammy (e.g., STOPH) has a greater probability of delaying naming than does a first-position whammy (e.g., PHOTs). The whammy in the first position will have a good chance of resolution by the time the last phoneme reaches critical activation; the whammy in the final position has an extremely poor chance of resolution before the previous phonemes reach critical activation.

Therefore, the DRC-L model predicts that the size of the whammy effect in nonword naming latency will depend on the left-to-right position of the whammied phoneme- just as the size of the regularity effect in word naming latency depends on the left-to-right position of the irregular GPC in an exception word (Coltheart \& Rastle, 1994; Rastle \& Coltheart, in press). Late whammies and early irregularities will be particularly harmful to naming. Both of these effects are due to the serial nature of the nonlexical route. 


\section{GENERAL DISCUSSION}

Our study of human nonword-naming latency has shown that fiveletter nonword strings with three graphemes have longer naming latencies than five-letter nonword strings with five graphemes. This result is also true of the DRC model when its nonlexical route operates letter by letter (rather than grapheme by grapheme). We therefore conclude that the relevant variable controlling the length effect on reading aloud is the letter, not the grapheme.

But we have discovered that this conclusion must be accompanied by taking into consideration the whammy effect. Because the unit of nonlexical translation is the letter, spurious competitive phoneme activations are generated for letter strings whenever a multiletter grapheme is submitted to the nonlexical route for translation. These spurious phoneme activations slow the rise of activation for the correct phonemes, and thus produce a latency cost. Even if there are no multiletter graphemes in the string, spurious phonemes can still be activated by single-letter graphemes if their translations depend on certain kinds of contextsensitive GPC or phonotactic rules.

The results presented here are inconsistent with an explanation at the level of articulation; if this difference in naming latency were due to late processes such as articulation, strings with few graphemes and thus few phonemes would most likely be pronounced more quickly than would strings with many graphemes. We observed exactly the opposite effect.

Our interpretation of the whammy effect depends crucially on the serial operation of the nonlexical route. It might be, however, that the whammy effect will occur in any model--serial or parallel-that maps letters to phonemes. Within this representational structure, when a multiletter grapheme such as PH occurs, the constituent letters of that grapheme activate inappropriate phonemes $(/ \mathrm{p} /$ and $/ \mathrm{h} /)$ as well as the correct phoneme $(/ \mathbf{f} /)$; competition between all of these phonemes could produce a whammy effect by slowing the rise of activation for the correct phoneme. On this account of the whammy effect, it is irrelevant whether the grapheme-to-phoneme translation process occurs serially or in parallel.

We have explored this issue briefly in a simulation using a system that operates in parallel, the attractor network implemented by Plaut et al. (1996). The nonwords used in the experiments reported here were submitted to the attractor network, which named 19/24 five-grapheme nonwords correctly and $17 / 24$ three-grapheme nonwords correctly (binomial test, $p=.72$ ). After errors were removed from the analysis, 14 matched pairs of items remained; for these items, three-grapheme items were named slightly more slowly $(M=1.88)$ than were five-grapheme items $(M=1.79)$, and this difference approached significance $[F(1,13)=$ $4.34, p=.058]$. Given the extremely high error rate of the model in reading these nonwords and the marginal significance of the main effect, it is unclear exactly what to conclude from these data. However, in an attempt to assess whether slightly slowed naming of the whammied items occurred in the network due to competition among multiple phoneme units activated by the individual letters of multiletter graphemes, it seemed worthwhile to analyze the final activations of all 61 phoneme units in the attractor network for each of the 14 three-grapheme items under consideration. We sought to determine whether, for example, the processing of SOACH by the network was slowed because the constituent letters of the $\mathrm{OA}$ and $\mathrm{CH}$ digraphs activated inappropriate phonemes that competed with correct phonemes activated by the digraph. Our analyses of the network revealed that the constituent letters of a digraph yielded inappropriate phoneme activations which were the most active of all spurious phoneme activations in only $27 \%$ of the cases in which this could occur over the 14 relevant items. The most active incorrect unit in SOACH was not one spuriously activated by the $\mathrm{OA}$ or $\mathrm{CH}$ digraph, but rather was the onset unit for $/ \mathrm{b} /$. Thus, if the attractor network produces a whammy effect at all, it is reasonably clear that the cause of the effect is not competition from the phonemes corresponding to the constituent letters of multiletter graphemes. Whether it is true in general that no purely parallel model of reading can generate a whammy effect remains, of course, to be determined, possibly by an experiment that directly examines the effect of the position of a whammy in a nonword

It has not escaped our attention that the whammy phenomenon has wide implications for any work in which the contributions of nonlexi- cal phonological recoding to reading are being explored-for example, work on lexical decision with pseudohomophonic nonwords (e.g., Coltheart, Davelaar, Jonasson, \& Besner, 1977) or work on semantic categorization with foils that are pseudohomophones of category members (e.g., Coltheart, Patterson, \& Leahy, 1994). Many would argue that, in both kinds of work, the effect of the pseudohomophone depends on its accessing the phonological representation of its base word. If so, then the DRC model predicts that the size of the pseudohomophone effect will be determined, in part, by the whammy phenomenon. CHEzE will have little or no harmful effect in lexical decision or in semantic categorization, because its double whammy will greatly slow activation of the phonological entry for CHEESE. On the other hand, BREDD-in the absence of any whammies - will be deadly.

\section{REFERENCES}

Coltheart, M., Curtis, B., Atkins, P., \& Haller, H. (1993). Modeis of reading aloud: Dual-route and parallel-distributed-processing approaches. Psychological Review, 100, 589-608.

Coltheart, M., Davelaar, E., Jonasson, J. T., \& Besner, D. (1977). Access to the internal lexicon. In S. Dornic (Ed.), Attention and performance $V I$ (pp. 535-555). Hillsdale, NJ: Erlbaum.

Coltheart, M., Langdon, R., \& Haller, M. (1996). Computational cognitive neuropsychology and acquired dyslexia. In B. Dodd, R. Campbell, \& L. Worrall (Eds.), Evaluating theories of language: Evidence from disordered communication (pp. 9-36). London: Whurr.

Coltheart, M., Patterson, K., \& Leahy, J. (1994). When a ROWS is a ROSE: Phonological effects in written word comprehension. $Q u a r$ terly Journal of Experimental Psychology, 47A, 917-955.

Coltheart, M., \& Rastle, K. (1994). Serial processing in reading aloud: Evidence for dual-route models of reading. Journal of Experimental Psychology: Human Perception \& Performance, 20, 1197 1211 .

Content, A., \& Peereman, R. (1992). Single and multiple process models of print to speech conversion. In J. Algeria, D. Holender, J. Junca de Morais, \& M. Radeau (Eds.), Analytic approaches to human cognition (pp. 213-236). Amsterdam: Elsevier.

Derouesne, J., \& Beauvois, M. F. (1979). Phonological processing in reading: Data from alexia. Journal of Neurology, Neurosurgery, \& Psychiatry, 42, 1125-1 132.

Forster, K. I., \& Forster, J. C. (1990). User's guide to the DMASTR display system: Laboratory software for mental chronometry. Unpublished manual.

Frederiksen, J. R., \& Kroll, J. F. (1976). Spelling and sound: Approaches to the internal lexicon. Journal of Experimental Psychology: Human Perception \& Performance, 2, 361-379.

HENDERSON, L. (1982). Orthography and word recognition in reading. London: Academic Press.

PAAP, K. R., \& NoEL, R. W. (1991). Dual-route models of print to sound: Still a good horse race. Psychological Research, 53, 13-24.

Plaut, D. C., McClelland, J. L., Seidenberg, M. S., \& PatrerSON, K. (1996). Understanding normal and impaired word reading: Computational principles in quasi-regular domains. Psychological Review, 103, 56-115.

RASTLE, K., \& COLTHEART, M. (in press). Serial and strategic effects in reading aloud. Journal of Experimental Psychology: Human Perception \& Performance.

Seidenberg, M. S., Waters, G. S., Barnes, M. A., \& Tanenhaus, M. K. (1984). When does irregular spelling or pronunciation influence word recognition? Journal of Verbal Learning \& Verbal Behavior, 23, 383-404.

WEEKES, B. S. (1997). Differential effects of number of letters on word and nonword naming latency. Quarterly Journal of Experimental Psychology, 50A, 439-456.

\section{NOTES}

1. The DRC-G model was built only to explore how a serially operating, grapheme-by-grapheme nonlexical procedure would behave for 
the purposes outlined here. We experienced considerable difficulty in trying to design an implementation of this nonlexical procedure, and the way it was finally implemented in the DRC-G model is relatively implausible: the nonlexical route of the DRC-G model first translates the entire letter string to a phoneme string via application of the GPC rules, then passes each phoneme in serial order to the phoneme system. If the human data suggest that the length effect is determined by the number of graphemes in a letter string and not by the number of letters in the string, then developing a suitable implementation of this nonlexical route may prove challenging.
2. The phoneme symbols used in this paper are $/ \mathrm{f} / \rightarrow \mathrm{EACE}, / \mathrm{Q} / \rightarrow$ POT, $/ \mathrm{u} / \rightarrow$ BOOT, $/$ p/ $\rightarrow$ PUT, $/ 1 / \rightarrow$ BAY,$/ 1 / \rightarrow$ LAST, $/ \mathrm{s} / \rightarrow \underline{\mathrm{SUN}}$, $\mid \mathrm{z} / \rightarrow$ ZAP, $/ \mathrm{N} / \rightarrow \mathrm{BANG}, / \mathbf{n} / \rightarrow$ NEST, $/ \mathrm{k} / \rightarrow$ KISS.

3. The stimulus-matching constraints that we adopted forced us to use many items with unique bodies. We are grateful to Ken Paap for suggesting to us that this property of the stimulus set may have encouraged a letter-by-letter translation strategy that would not be utilized if body information were present. We acknowledge this possibility; whether the whammy effect persists in nonwords with frequently occurring bodies remains an empirical question.

APPENDIX

Human and Simulation Data

\begin{tabular}{|c|c|c|c|c|c|c|c|}
\hline $\begin{array}{l}\text { Three- } \\
\text { Grapheme } \\
\text { Nonword }\end{array}$ & RT (msec) & $\begin{array}{c}\text { DRC-G } \\
\text { RT } \\
\text { (cycles) }\end{array}$ & $\begin{array}{c}\text { DRC-L } \\
\text { RT } \\
\text { (cycles) }\end{array}$ & $\begin{array}{c}\text { Five- } \\
\text { Grapheme } \\
\text { Nonword }\end{array}$ & RT (msec) & $\begin{array}{c}\text { DRC-G } \\
\text { RT } \\
\text { (cycles) }\end{array}$ & $\begin{array}{c}\text { DRC-L } \\
\text { RT } \\
\text { (cycles) }\end{array}$ \\
\hline BARCH & 547 & 128 & 161 & BREPS & 583 & 157 & 157 \\
\hline BERSH & 578 & 131 & 169 & BULSK & 578 & 157 & 157 \\
\hline BOACE & 607 & - & 169 & BLUSP & 601 & - & 157 \\
\hline $\mathrm{SOACH}$ & 523 & 129 & 163 & STELD & 523 & 155 & 155 \\
\hline FOUSH & 607 & 131 & 167 & FENKS & 572 & 157 & 157 \\
\hline KEESH & 534 & 125 & 164 & KELST & 543 & 157 & 157 \\
\hline DOATH & 566 & 122 & 148 & DELST & 569 & 157 & 157 \\
\hline DOAPH & 649 & 132 & 170 & DALST & 574 & 157 & 157 \\
\hline DOISH & 577 & 132 & 170 & DRUSP & 557 & 157 & 157 \\
\hline FEECH & 589 & 131 & 166 & FREPS & 574 & 157 & 157 \\
\hline GAIFF & 575 & 132 & 157 & GLECT & 616 & 157 & 157 \\
\hline FOOCE & 575 & 190 & 152 & FRULS & 602 & 157 & 185 \\
\hline GAICH & 579 & 132 & 170 & GLUPT & 587 & 157 & 157 \\
\hline FOOPH & 698 & 132 & 170 & FROLP & 598 & 152 & 152 \\
\hline SERCE & 526 & - & 167 & STRIK & 504 & - & 139 \\
\hline GAUCH & 642 & 132 & 169 & GLESP & 599 & 157 & 157 \\
\hline VERCK & 627 & 132 & 157 & VULSK & 565 & 157 & 157 \\
\hline DARCH & 538 & 128 & 161 & DREPS & 508 & 152 & 152 \\
\hline TAWSH & 585 & 132 & 170 & TWENK & 532 & 157 & 170 \\
\hline TAIVE & 562 & 132 & 157 & TRUSP & 525 & 149 & 149 \\
\hline GOICH & 611 & 132 & 170 & GLOSP & 592 & 152 & 152 \\
\hline PAISH & 561 & 132 & 170 & PRENK & 562 & 157 & 170 \\
\hline GHOAN & 623 & 131 & 157 & GRUSK & 571 & 157 & 157 \\
\hline $\mathrm{KIRCH}$ & 572 & 131 & 169 & KRELM & 588 & 157 & 157 \\
\hline
\end{tabular}

(Manuscript received May 29, 1997;

revision accepted for publication October $30,1997$. 